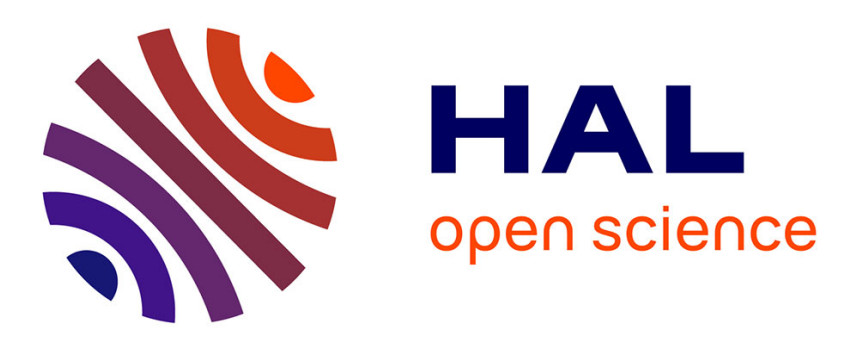

\title{
Order-disorder or phase-separation transition: Analysis of the Au-Pd system by the effective site energy model
}

F. Berthier, J. Creuze, T. Gabard, B. Legrand, M.-C. Marinica, C. Mottet

\section{To cite this version:}

F. Berthier, J. Creuze, T. Gabard, B. Legrand, M.-C. Marinica, et al.. Order-disorder or phaseseparation transition: Analysis of the Au-Pd system by the effective site energy model. Physical Review B: Condensed Matter and Materials Physics (1998-2015), 2019, 99 (1), 10.1103/PhysRevB.99.014108 . hal-02357985

\section{HAL Id: hal-02357985 \\ https://hal.science/hal-02357985}

Submitted on 11 Nov 2019

HAL is a multi-disciplinary open access archive for the deposit and dissemination of scientific research documents, whether they are published or not. The documents may come from teaching and research institutions in France or abroad, or from public or private research centers.
L'archive ouverte pluridisciplinaire HAL, est destinée au dépôt et à la diffusion de documents scientifiques de niveau recherche, publiés ou non, émanant des établissements d'enseignement et de recherche français ou étrangers, des laboratoires publics ou privés. 


\title{
Order-disorder or phase-separation transition: Analysis of the Au-Pd system by the effective site energy model
}

\author{
F. Berthier ${ }^{1,2}$ J. Creuze, ${ }^{2}$ T. Gabard, ${ }^{2}$ B. Legrand, ${ }^{3}$ M.-C. Marinica, ${ }^{3}$ and C. Mottet $^{4}$ \\ ${ }^{1}$ Centre National de la Recherche Scientifique, UMR 8182, F91405 Orsay Cedex, France \\ ${ }^{2}$ ICMMO/SP2M, Université Paris Sud, UMR 8182, Université Paris-Saclay, F91405 Orsay Cedex, France \\ ${ }^{3}$ CEA, DEN, Service de Recherches de Métallurgie Physique, Université Paris-Saclay, F91191 Gif-sur-Yvette Cedex, France \\ ${ }^{4}$ Aix-Marseille Université, Centre National de la Recherche Scientifique, CINaM UMR7325, Campus de Luminy, F13288 Marseille, France
}

(Received 5 July 2018; revised manuscript received 15 December 2018; published 15 January 2019)

\begin{abstract}
Using a many-body interatomic potential for the Au-Pd system, we determine a bulk phase diagram which presents unexpected characteristics for a system with ordering tendency. Indeed, this system displays a miscibility gap between pure $\mathrm{Pd}$ and $\mathrm{Au}_{c} \mathrm{Pd}_{1-c}$ (with $c \approx 0.2$ ) beyond the order/disorder critical temperature of the $\mathrm{AuPd}_{3}$ compound. Enthalpic and entropic contributions of the permutation free energy are determined via Monte Carlo simulations, in particular the vibrational entropy, which is in good agreement with direct calculation. Finally, the effective site energy model, recently developed to describe the thermodynamical forces driving the bulk phase diagram, is used to demonstrate that the miscibility gap in the Au-Pd system comes from competition between elastic and chemical effects.
\end{abstract}

DOI: 10.1103/PhysRevB.99.014108

\section{INTRODUCTION}

Theoretical predictions of bulk phase diagrams pave the way for studies of more complex phase diagrams such as those for alloy surfaces or nanoalloys. Up to now, the theoretical studies have been mainly performed using $a b$ initio calculations on a large number of ordered structures at different concentrations. In this framework, cluster expansion (CE) method $[1,2]$ allows one to extract two-body (or more) interactions for each concentration and, subsequently, the phase diagram is obtained via statistical physics methods such as cluster variation method [3,4] and Monte Carlo (MC) simulations.

In the systems with low misfit these approaches are powerful. However, when applied for systems with a large difference in lattice parameter between the two constituents, they can be less efficient and accurate. The atomic relaxations play an important role. In such a case, the elastic effect (also called "size effect") is coupled to the chemical effect and can lead to important variations of the $\mathrm{CE}$ interactions as a function of the concentration. Moreover, these interactions deduced from ordered structures can be misleading for disordered structures. Finally, these methods are hardly transferable to more complex crystallographic structures, such as surfaces or nanoalloys, because interactions must be calculated between all inequivalent sites and for all concentration profiles.

This paper reveals an alternative way to analyze phase diagrams of systems with large lattice mismatch. Using interatomic potentials derived from the tight-binding formalism within the second moment approximation (SMA) of the density of states, we perform atomistic Monte Carlo simulations with atomic displacements to get isotherms $\Delta \mu(c)$, i.e., the difference of chemical potentials between the two constituents as a function of the concentration $c$ considering the system $\mathrm{A}_{c} \mathrm{~B}_{1-c}$. Then we analyze the enthalpic and entropic as well as configurational as vibrational components of $\Delta \mu(c)$, which constitute the permutation free enthalpy corresponding to the permutation of an atom of type B into an atom of type A. The most original part of this paper is the use of the effective site energy (ESE) formalism [5] to decompose the permutation enthalpy into chemical and elastic contributions. This analysis provides a better understanding of the interplay between competing short-range order (SRO) and long-range order interactions which can be at the origin of complex phase diagrams. Such effects are also the topic of numerous studies in the field of spin-crossover materials [6-15].

To illustrate this theoretical approach, we chose the AuPd system, which has been often studied in particular for its catalytic activity, notably in the CO oxidation [16]. However, the knowledge of its bulk phase diagram remains under debate (Fig. 1).

At high temperature, the Au-Pd alloy forms a solid solution with the fcc structure on the whole range of concentration [17]. At low temperature, the authors assumed the existence of $\mathrm{AuPd}_{3}$ and $\mathrm{Au}_{3} \mathrm{Pd}$ ordered phases with the $L 1_{2}$ structure and the AuPd one with the $L 1_{0}$ structure. Dashed lines in Fig. 1 are purely speculative. These ordered phases have not been observed in bulk alloys but on thin films grown by metallic evaporation on $\mathrm{NaCl}$ or $\mathrm{KCl}$ monocrystals and high-temperature annealing [18-21]. It can thus be suspected that elastic effects have a significant role to play. On the other hand, the differences in the hierarchy of the order/disorder critical temperatures are rather astonishing: whereas the critical temperature of the $L 1_{2}$ structure is equal to $870^{\circ} \mathrm{C}$ for $\mathrm{AuPd}_{3}$ and $850^{\circ} \mathrm{C}$ for $\mathrm{Au}_{3} \mathrm{Pd}$, that of the $L 1_{0}$ structure is only of the order of $100^{\circ} \mathrm{C}$. This contrasts strongly with the fact that the critical temperature of the $L 1_{0}$ phase is usually slightly higher than those of the $L 1_{2}$ phases. The very existence of the $L 1_{0}$ phase for the Au-Pd alloy is questioned [19], this structure being unfavorable as compared to $\mathrm{Au}_{2} \mathrm{Pd}_{2}$ 


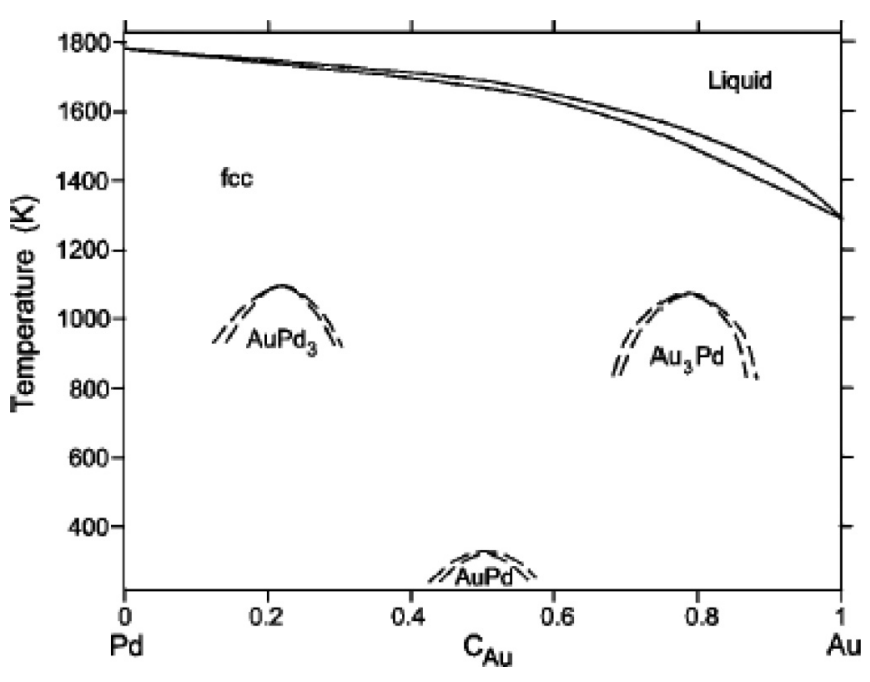

FIG. 1. Experimental Au-Pd phase diagram (from Ref. [13]). Dashed lines are purely speculative.

structure in ab initio calculations [22-24]. More recently, the ordered phases of nanoparticles have been observed [25] but at different temperatures than those of the bulk phases [25]. Low critical temperatures have also been obtained by Monte Carlo simulations using embedded atom method [26,27]. Thus AuPd phase diagram remains controversial.

The approach we propose here will be particularly well adapted to treat the coupling between a size effect $(\Delta r / r=$ $6 \%$ ) and a weak chemical ordering effect, and will allow us to elucidate the origin of a very astonishing topology of the phase diagram: the Pd-rich side of the system displays a miscibility gap which is unexpected for an alloy with an ordering tendency.

The paper is organized as follows. In Sec. II, the free permutation and mixing enthalpy of the $\mathrm{Au}-\mathrm{Pd}$ system are characterized in the whole range of composition together with an analysis of the different enthalpic and entropic contributions. Section III presents the site energy approach and the analysis of the mixing and permutation enthalpies in terms of elastic and chemical contributions. The phase diagram is described and analyzed in Sec. IV, before the conclusion.

\section{FREE PERMUTATION AND MIXING ENTHALPY OF $\mathrm{Au}_{c} \mathbf{P d}_{1-c}$}

\section{A. Methodology}

MC simulations with atomic relaxations in the canonical ensemble (see Appendix A) allow determining the equilibrium chemical potential difference $\Delta \mu$ as a function of both the concentration $c$ of the alloy $\mathrm{A}_{c} \mathrm{~B}_{1-c}$ and the temperature. Such a purely numerical method does not lead to analytical equations, which makes it difficult to analyze the results. To overcome this disadvantage and characterize the driving forces piloting the bulk phase diagram of the $\mathrm{Au}_{c} \mathrm{Pd}_{1-c}$ system, we develop a scheme coupling analytical methods on lattice approaches (mean field approximation for the Ising model or effective site energy formalism) and Monte Carlo simulations with atomic displacements.

The difference in equilibrium chemical potential $\Delta \mu(c)$ mentioned before corresponds to the free enthalpy bal- ance during a permutation ${ }^{1}$ of one $\mathrm{Pd}$ atom into one $\mathrm{Au}$ atom, which we call further the free permutation enthalpy $\Delta G_{\mathrm{SMA}}^{\mathrm{perm}}(c)$. This quantity can be decomposed as $\Delta G_{\mathrm{SMA}}^{\mathrm{perm}}=\Delta H_{\mathrm{SMA}}^{\mathrm{perm}}-T \Delta S_{\mathrm{SMA}}^{\text {perm }}$, where $\Delta H_{\mathrm{SMA}}^{\text {perm }}$ and $\Delta S_{\mathrm{SMA}}^{\text {perm }}$ are the enthalpic and entropic balances during the permutation of one $\mathrm{Pd}$ atom into one $\mathrm{Au}$ atom.

In standard mean field approximation (MFA), on a rigid lattice, the isotherms are written as

$$
\Delta \mu^{\mathrm{MFA}}=\Delta H_{\mathrm{RSS}}^{\mathrm{perm}}+k_{B} T \ln \frac{c}{1-c},
$$

where $\Delta H_{\mathrm{RSS}}^{\mathrm{perm}}$ represents the permutation enthalpy for a random solid solution (RSS) and

$$
\Delta S_{\mathrm{RSS}}^{\mathrm{perm}}=-k_{B} \ln \frac{c}{1-c}
$$

its configurational permutation entropy. This relation describes the evolution of $\Delta \mu$ as a function of $c$ for a random solid solution without taking into account any local ordering or atomic vibrations. To get a full analysis of the Monte Carlo simulations including atomic displacements, the relation (1) has to be extended to other contributions:

$$
\Delta G_{\mathrm{SMA}}^{\mathrm{perm}}=\Delta G_{\mathrm{RSS}}^{\mathrm{perm}}+\Delta G_{\mathrm{SRO}}^{\mathrm{perm}}+\Delta G_{\mathrm{vib}}^{\mathrm{perm}},
$$

where the following hold.

(1) $\Delta G_{\mathrm{RSS}}^{\mathrm{perm}}$ is the permutation free enthalpy of a RSS.

(2) $\Delta G_{\mathrm{SRO}}^{\mathrm{perm}}$ is the permutation free enthalpy excess due to SRO. Even above the critical order/disorder temperature, characterized by the disappearance of the long-range order, SRO remains, which modifies the permutation free enthalpy. The higher the temperature, the less the site occupation is correlated, and $\Delta G_{\mathrm{SRO}}^{\text {perm }}$ tends to zero at very high temperature.

(3) $\Delta G_{\text {vib }}^{\text {perm }}$ is the vibrational permutation free enthalpy due to the atomic displacements in the Monte Carlo simulations.

Each of these free enthalpy contributions is the sum of one enthalpic and one entropic term $(\Delta G=\Delta H-T \Delta S)$, except for the vibrational permutation enthalpy, which is zero above the Debye temperature $\left(\Delta H_{\mathrm{vib}}^{\text {perm }}=0\right)$ :

$$
\begin{aligned}
\Delta H_{\mathrm{SMA}}^{\text {perm }} & =\Delta H_{\mathrm{RSS}}^{\text {perm }}+\Delta H_{\mathrm{SRO}}^{\text {perm }} \\
\Delta S_{\mathrm{SMA}}^{\text {perm }} & =\Delta S_{\mathrm{RSS}}^{\text {perm }}+\Delta S_{\mathrm{SRO}}^{\text {perm }}+\Delta S_{\mathrm{vib}}^{\text {perm }} .
\end{aligned}
$$

All these contributions will be extracted from the Monte Carlo simulations within the semiempirical potentials as follows.

\section{Determination of $\Delta H_{\mathrm{SMA}}^{\mathrm{perm}}$ and decomposition}

The permutation enthalpy is directly determined from the Monte Carlo simulations in the canonical ensemble:

$$
\Delta H_{\mathrm{SMA}}^{\mathrm{perm}}(c)=\frac{1}{N_{\mathrm{at}}} \frac{\partial E_{\mathrm{SMA}}^{\mathrm{tot}}(c)}{\partial c},
$$

$N_{\text {at }}$ being the total number of atoms in the simulation box and $E_{\mathrm{SMA}}^{\text {tot }}(c)$ being the energy of the simulation box for the

\footnotetext{
${ }^{1}$ It would be more accurate to use the term transmutation rather than permutation to describe the nature of species changes by exchange with an external reservoir.
} 
composition $c . \Delta H_{\mathrm{RSS}}^{\mathrm{perm}}(c)$ comes from [Eq. (5)] applied to $E_{\mathrm{RSS}}^{\text {tot }}$ for a random solid solution. Then $\Delta H_{\mathrm{SRO}}^{\mathrm{perm}}$ is simply deduced from Eq. (4a): $\Delta H_{\mathrm{SRO}}^{\mathrm{perm}}=\Delta H_{\mathrm{SMA}}^{\mathrm{perm}}-\Delta H_{\mathrm{RSS}}^{\mathrm{perm}}$.

\section{Determination of $\Delta S_{\mathrm{SMA}}^{\mathrm{perm}}$ and decomposition}

The permutation entropy $\Delta S_{\mathrm{SMA}}^{\mathrm{perm}}$ is obtained from the calculation of each of its components [Eq. (4b)]. $\Delta S_{\mathrm{RSS}}^{\text {perm }}$ is directly determined by the mean field expression (2). $\Delta S_{\mathrm{SRO}}^{\mathrm{perm}}$ is calculated using the formula of pair probabilities $[28,29]$, given in Appendix B. Its expression depends on the SRO parameter $\eta$ which is determined from the Monte Carlo simulations in the canonical ensemble. We used the Warren-Cowley parameter [30] $\eta=1-P^{\mathrm{BA}} / c$ where $P^{\mathrm{BA}}$ is the probability to find atoms of type $\mathrm{A}$ as first neighbors of one B atom. Therefore, for a fully disordered alloy, there is no correlation between $\mathrm{A}$ and $\mathrm{B}$ atoms and $\eta=0$. For a system with ordering tendency, $\eta<0$, whereas for a system with demixtion tendency $\eta>0$. The last component is the vibrational permutation entropy, which is directly obtained from $S_{\text {vib }}^{\text {tot }}(c)$ (see Appendix A 4) by differentiating Eq. (A2) with respect to the concentration [similarly to Eq. (5)].

It is worth noticing that for any permutation quantity $\Delta X^{\text {perm }}$ (either enthalpic or entropic) it is possible to get the equivalent mixing quantity $\Delta X^{\text {mixing }}$ via the following equation:

$$
\Delta X^{\mathrm{mixing}}(c)=\int_{0}^{c} \Delta X^{\mathrm{perm}}(u) d u-c \int_{0}^{1} \Delta X^{\mathrm{perm}}(u) d u .
$$

\section{B. Results}

We present in this part the results concerning the permutation free enthalpy of $\mathrm{Au}_{c} \mathrm{Pd}_{1-c}$ in the whole range of concentration $c$ as obtained by the Monte Carlo simulations and its decomposition in terms of enthalpic, configurational, and vibrational entropic contributions. Then we deduce the mixing free enthalpy of the system with the same analysis, leading to a nice characterization of the energetics and thermodynamics of this system.

Figure 2 shows the $\Delta \mu(c)$ isotherm coming from the Monte Carlo simulations at $T=500 \mathrm{~K}$. The isotherm is monotonously increasing on the whole range of concentration.

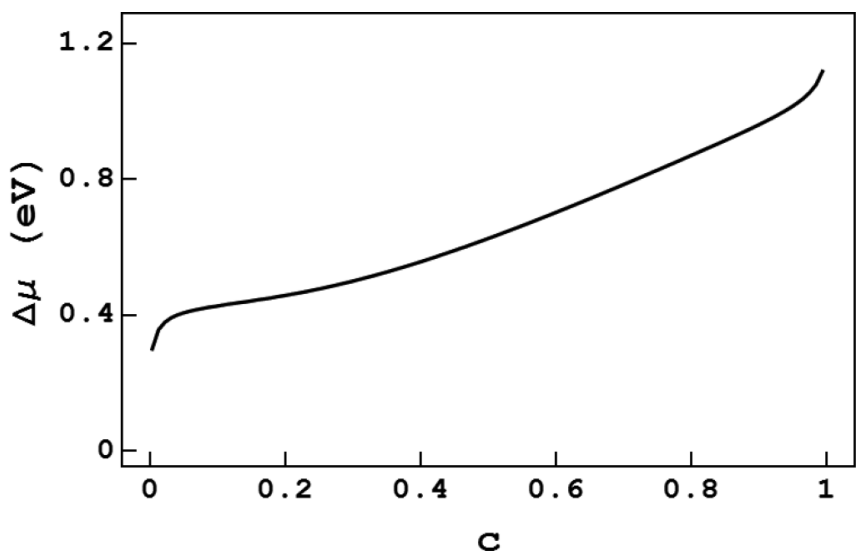

FIG. 2. Evolution of $\Delta \mu$ as a function of the concentration $c$ at $T=500 \mathrm{~K}$ from Monte Carlo SMA simulations.

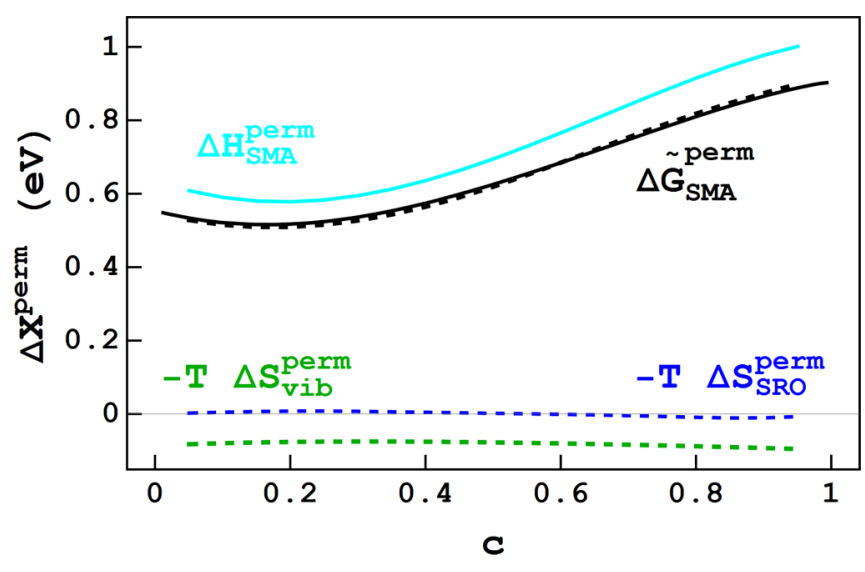

FIG. 3. Evolution as a function of the concentration $c$ at $T=500 \mathrm{~K}$ of $\Delta H_{\mathrm{SMA}}^{\mathrm{perm}}$ (cyan), $-T \Delta S_{\mathrm{SRO}}^{\mathrm{perm}}$ (dashed blue), $-T \Delta S_{\text {vib }}^{\text {perm }}$ (dashed green), $\Delta \tilde{G}_{\mathrm{SMA}}^{\text {perm }} \quad$ (black), and $\Delta H_{\mathrm{SMA}}^{\mathrm{perm}}-T\left(\Delta S_{\mathrm{SRO}}^{\text {perm }}+\Delta S_{\mathrm{vib}}^{\mathrm{perm}}\right)($ dashed black $)$.

It represents the free permutation enthalpy that we decompose in enthalpic and entropic contributions.

From the $\Delta \mu(c)$ isotherm (Fig. 2), we extract the quantity $\Delta \tilde{G}_{\mathrm{SMA}}^{\text {perm }}(c)$, which we define as the permutation free enthalpy, from which we subtract the disordered solid solution entropy $\Delta \tilde{G}_{\mathrm{SMA}}^{\text {perm }}(c)=\Delta G_{\mathrm{SMA}}^{\mathrm{perm}}(c)+T \Delta S_{\mathrm{RSS}}^{\mathrm{perm}}(c)$ with $\Delta S_{\mathrm{RSS}}^{\mathrm{perm}}(c)$ given by Eq. (2).

Thereby, this quantity and the permutation enthalpy $\Delta H_{\mathrm{SMA}}^{\mathrm{perm}}(c)$ differ only by the SRO and vibrational entropic contributions. $\Delta H_{\mathrm{SMA}}^{\mathrm{perm}}$ is plotted in Fig. 3 together with the $\mathrm{SRO}$ and vibrational entropies $\left(-T \Delta S_{\mathrm{SRO}}^{\text {perm }}\right.$ and $\left.-T \Delta S_{\mathrm{vib}}^{\text {perm }}\right)$. We can notice that $\Delta H_{\mathrm{SMA}}^{\text {perm }}(c)$ is not monotonic whereas in standard models (Ising models in mean field approximation) we would expect a linear increasing variation for systems with ordering tendency such as Au-Pd. We will see in the following that this point has important consequences for the phase diagram, and the origin of this nonmonotonic behavior of $\Delta H_{\mathrm{SMA}}^{\text {perm }}(c)$ will be understood thanks to the site energy model developed in the next section.

We notice also in Fig. 3 that the entropic contributions are weak (the SRO one is even negligible) and their variations with concentration are also very weak. Therefore, the two curves $\Delta H_{\mathrm{SMA}}^{\mathrm{perm}}(c)$ and $\Delta \tilde{G}_{\mathrm{SMA}}^{\mathrm{perm}}(c)$ are almost parallel. Finally, the agreement between $\Delta \tilde{G}_{\mathrm{SMA}}^{\text {perm }}(c)$ and its reconstitution by $\Delta H_{\mathrm{SMA}}^{\text {perm }}-T\left(\Delta S_{\mathrm{SRO}}^{\text {perm }}+\Delta S_{\mathrm{vib}}^{\text {perm }}\right) \quad$ is remarkable, which proves the relevance of the analysis.

The related mixing quantities are plotted in Fig. 4 using Eq. (6). As expected for a system with ordering tendency, the mixing enthalpy is negative in the whole concentration range. The SRO and vibrational entropic contributions are positive but weak. As with the permutation free enthalpy, the mixing free enthalpy is perfectly reconstituted with the different components of the SRO and vibrational entropies.

\section{DRIVING FORCES ANALYSIS USING THE ESE MODEL}

\section{A. ESE model}

The ESE model has been described previously for Au$\mathrm{Ni}$ and $\mathrm{Ag}-\mathrm{Cu}$ systems [5]. It enables us to characterize 


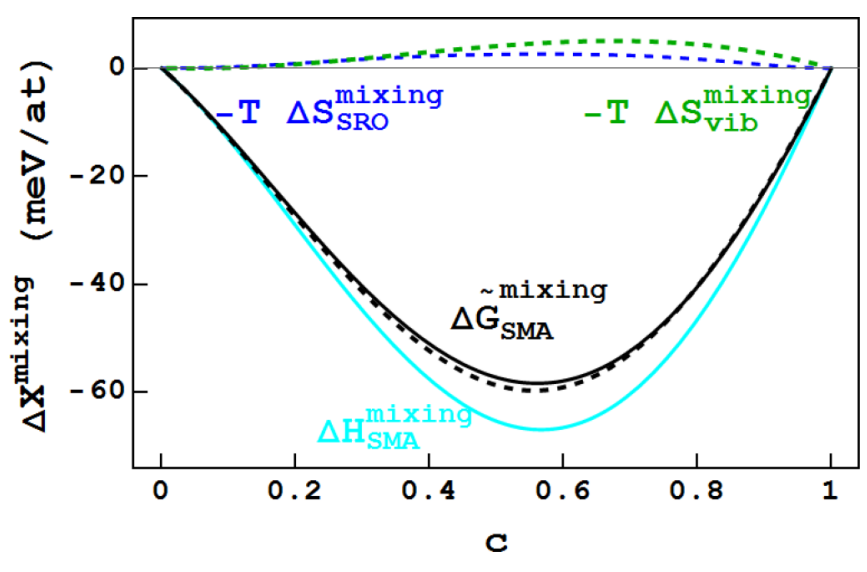

FIG. 4. Evolution as a function of the concentration $c$ at $T=500 \mathrm{~K}$ of $\Delta H_{\mathrm{SMA}}^{\text {mixing }}$ (cyan), $-T \Delta S_{\mathrm{SRO}}^{\text {mixing }}$ (dashed blue), $-T \Delta S_{\text {vib }}^{\text {mixing }}$ (dashed green), $\Delta \tilde{G}_{\mathrm{SMA}}^{\text {mixing }}$ (black), and $\Delta H_{\mathrm{SMA}}^{\text {mixing }}-$ $T\left(\Delta S_{\mathrm{SRO}}^{\text {mixing }}+\Delta S_{\mathrm{vib}}^{\text {mixing }}\right)$ (dashed black).

the effective pair or triplet interactions taking into account atomistic relaxations and their variations with the nominal concentration. It requires an atomistic model to determine the energy of each inequivalent site as a function of the local and global concentration. We used the SMA model described in Appendix A 1. The site energies $E_{X}^{p}(c)(X=\mathrm{Au}, \mathrm{Pd})$ are calculated on a random solid solution of the alloy $\mathrm{Au}_{c} \mathrm{Pd}_{1-c}$ (see Appendix A 2) and depend on the chemical nature of the atom at the considered site, on its local chemical environment defined by $p$ (number of first neighbors of Au type among the 12 first neighbors in the fcc structure), and finally on the concentration $c$. They are presented in Fig. 5 for different values of the concentration of the random solid solution. The cohesive energy of Pd [Fig. 5(b)] is obtained for $c=0$ and $p=0$, and corresponds to $E_{\mathrm{Pd}}^{0}(0)=-4.98 \mathrm{eV}$, whereas that of $\mathrm{Au}$ [Fig. 5(a)], for $c=1$ and $p=12$, corresponds to $E_{\mathrm{Au}}^{12}(1)=-4.24 \mathrm{eV}$. Gold being less cohesive than palladium, the bigger the number $p$ of first neighbors of Au type,

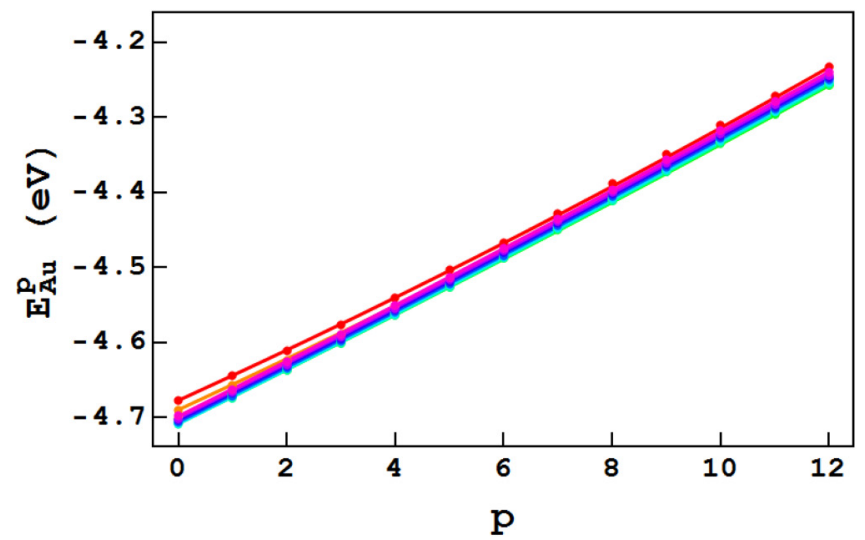

(a) the less cohesive the site. For a given concentration, the energy of one gold atom varies almost linearly with $p$, the curvature being slightly positive [Fig. 5(a)]. For a given $p$, the gold atom energy varies very slightly with the concentration [Fig. 5(a)]. Concerning $\mathrm{Pd}$ atoms, the energy variation with $p$ is much less than for $\mathrm{Au}(+0.2 \mathrm{eV}$ for $\mathrm{Pd}$ as compared to $+0.5 \mathrm{eV}$ for $\mathrm{Au}$ ) but the variation amplitude with concentration is larger, mainly for the small values of $p$ [Fig. 5(b)], and the curvature is slightly negative, i.e., an opposite sign as compared to Au.

We applied the effective site energy model by using Monte Carlo simulations on an effective rigid lattice to determine the $\Delta \mu(c)$ isotherms at different temperatures in the canonical ensemble (see Appendix A 3). This difference of chemical potential characterizes a new permutation free enthalpy for an effective rigid lattice $\Delta \mu=\Delta G_{\mathrm{ESE}}^{\mathrm{perm}}$, although on a rigid lattice these simulations take into account the elastic effects coming from the dependence of the site energies on the concentration. On the other hand, unlike Monte Carlo SMA simulations, they do not take into account atomic vibrations. The difference between the permutation free enthalpy with atomic relaxations coming from the Monte Carlo SMA simulations and that on the effective rigid lattice allows us to deduce the vibrational contribution $\Delta S_{\mathrm{vib}}^{\mathrm{perm}}=-\left(\Delta G_{\mathrm{SMA}}^{\mathrm{perm}}-\Delta G_{\mathrm{ESE}}^{\mathrm{perm}}\right) /\left(k_{B} T\right)$. This quantity, plotted in Fig. 6, is in good agreement with the direct calculation of the vibrational entropy $\Delta S_{\mathrm{RSS}}^{\text {perm }}(c)$ (see Appendix A 4), which gives confidence in the effective site energy model.

\section{B. Decomposition of the mixing and the permutation enthalpies within the ESE model}

By using the effective site energy model we reveal in this section the driving forces describing the bulk phase diagram. For that purpose the permutation enthalpy is split in three components, each of them related to three effects: cohesion, size, and chemistry. Each of them is developed in terms of site energies. The Hamiltonian of a random solid solution can be written as a function of the site energies in a combinatorial

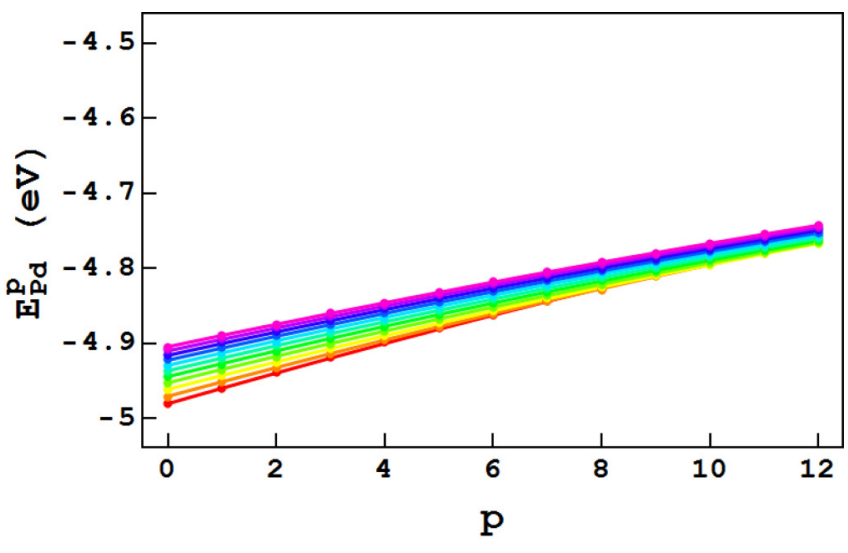

0.5 1

(b)

FIG. 5. Site energy of an atom $X=\mathrm{Au}, \mathrm{Pd}$ as a function of the number $p$ of Au neighbors, for different values of the concentration in $\mathrm{Au}_{c} \mathrm{Pd}_{1-c}$ alloy: $I=\mathrm{Au}(\mathrm{a})$ and $\mathrm{Pd}(\mathrm{b})$. The color scales between red ( $c=0$, Pd-pure system) and purple ( $c=1$, Au-pure system). The lines are simply a guide for the eyes. 


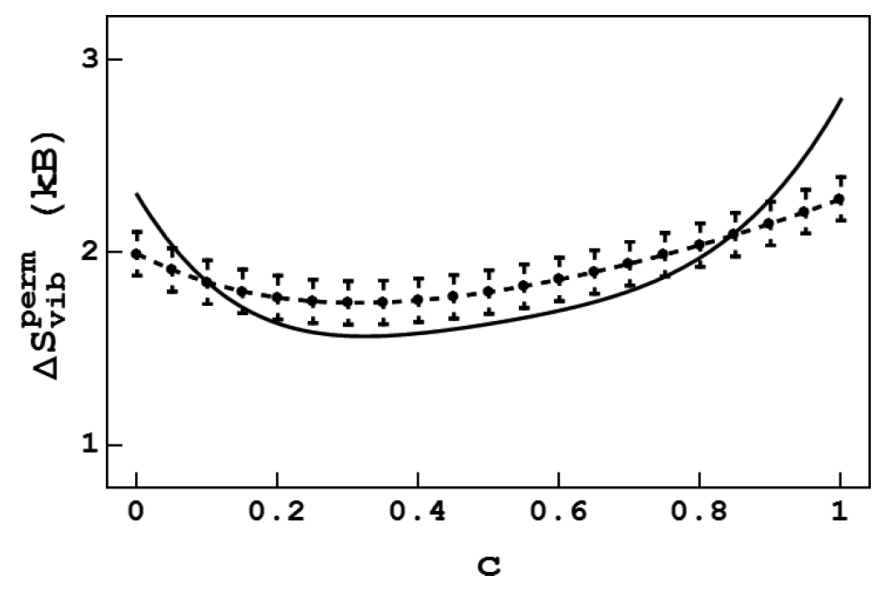

FIG. 6. Evolution as a function of the nominal concentration of $-\left(\Delta G_{\mathrm{SMA}}^{\mathrm{perm}}-\Delta G_{\mathrm{RR}}^{\mathrm{perm}}\right) / T$ from Monte Carlo simulations (full line) and of $\Delta S_{\text {vib }}^{\text {perm }}$ coming from direct calculations on random solid solutions (dashed lines) with error bars corresponding to the dispersion according to different RSS. All calculations are performed at $T=500 \mathrm{~K}$.

way [5]:

$$
H(c)=N_{\text {at }} \sum_{p=0}^{Z} C_{Z}^{p} c^{p}(1-c)^{Z-p}\left[c E_{A}^{p}(c)+(1-c) E_{B}^{p}(c)\right] .
$$

$C_{Z}^{p}$ are the binomial coefficients, i.e., the number of ways that $p$ A neighbors can be chosen among $Z$ neighbors disregarding their order. For a given concentration, the site energies calculated in the previous section can be expressed as a sum of a linear term in $p$, the most important one according to the Ising model, and a quadratic term in $p$ :

$$
E_{X}^{p}(c)=E_{X}^{0}(c)+\frac{p}{Z}\left[E_{X}^{Z}(c)-E_{X}^{0}(c)\right]+\varkappa_{X}(c) p(Z-p) .
$$

In Fig. 7, we give a schematic representation of this decomposition.

The linear Ising term corresponds to lattice pair interactions (see Appendix C) with a noticeable specificity: these interactions depend here on the nominal concentration via the elastic effect [5].

The quadratic term represents the excess energy as compared to pair interactions and can be considered as triplets contribution (see Appendix C). The curvatures $\varkappa_{X}$ with $X=$ $\mathrm{A}$ or B depend also on $c$, because of elastic effects.

Hence, the Hamiltonian of Eq. (7) can be written as two terms:

$$
H(c)=H_{\text {Ising }}(c)+H_{\text {triplets }}(c),
$$

where the Ising term is expressed as follows using Eqs. (7) and (8):

$$
\begin{aligned}
H_{\text {Ising }}(c) / N_{\text {at }}= & E_{B}^{0}(c)+\left[E_{A}^{Z}(c)-E_{B}^{0}(c)\right] c \\
& -c(1-c)\left\{\left[E_{A}^{Z}(c)-E_{A}^{0}(c)\right]\right. \\
& \left.-\left[E_{B}^{Z}(c)-E_{B}^{0}(c)\right]\right\} .
\end{aligned}
$$

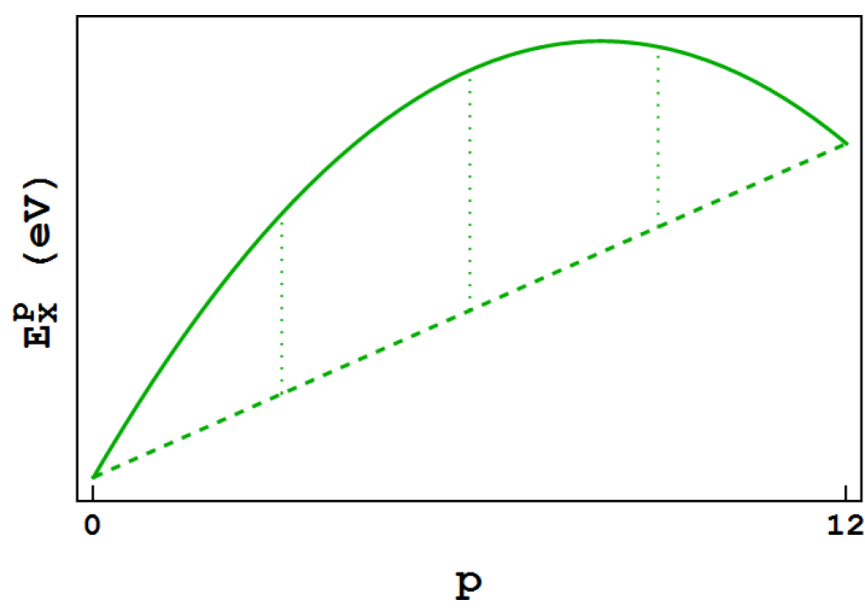

FIG. 7. Schematic representation of the $E_{X}^{p}$ variations (in green) as a function of $p$ for a given concentration. The linear part (Ising model) is represented by the dashed green line. The gap between $E_{X}^{p}$ and the Ising model for a given $p$ corresponds to the complementary quadratic term (dotted green lines).

We recognize the constant, linear, and quadratic terms of the usual Ising Hamiltonian [see Eqs. (C3) and (C8)].

The triplets term is expressed as a function of the two curvatures $\varkappa_{A}(c)$ and $\varkappa_{B}(c)$ (see Appendix $\mathrm{C}$ for more details):

$H_{\text {triplets }}(c) / N_{\text {at }}=Z(Z-1) c(1-c)\left[c \varkappa_{A}(c)+(1-c) \varkappa_{B}(c)\right]$.

We deduce from these expressions the mixing enthalpies of the random solid solution written as $\Delta H_{\mathrm{RSS}}^{\text {mixing }}(c)=H(c)-$ $\left[c E_{A}^{Z}(1)+(1-c) E_{B}^{0}(0)\right]$ in terms of site energies and its decomposition into three terms including size effect:

$$
\Delta H_{\mathrm{RSS}}^{\text {mixing }}(c)=\Delta H_{\mathrm{Ising}}^{\text {mixing }}(c)+\Delta H_{\text {triplets }}^{\text {mixing }}(c)+\Delta H_{\text {Size }}^{\text {mixing }}(c),
$$

where

$$
\begin{aligned}
\Delta H_{\text {Ising }}^{\text {mixing }}(c)= & -c(1-c)\left\{\left[E_{A}^{Z}(c)-E_{A}^{0}(c)\right]\right. \\
& \left.-\left[E_{B}^{Z}(c)-E_{B}^{0}(c)\right]\right\}, \\
\Delta H_{\text {triplets }}^{\text {mixing }}(c)= & Z(Z-1) c(1-c)\left[c \varkappa_{A}(c)\right. \\
& \left.+(1-c) \varkappa_{B}(c)\right], \\
\Delta H_{\text {Size }}^{\text {mixing }}(c)= & c\left[E_{A}^{Z}(c)-E_{A}^{Z}(1)\right] \\
& +(1-c)\left[E_{B}^{0}(c)-E_{B}^{0}(0)\right] .
\end{aligned}
$$

The terms $E_{A}^{Z}(c)-E_{A}^{Z}(1)$ and $E_{B}^{0}(c)-E_{B}^{0}(0)$ in [Eq. (12c)] correspond, respectively, for sites occupied by A or B atoms to the variation of site energies with the nominal concentration [as compared to pure metals $E_{A}^{Z}(1)$ and $\left.E_{B}^{0}(0)\right]$ for the same local environment, i.e., for $\mathrm{Z}(=12)$ A neighbors for $\mathrm{A}$ atoms (a cluster of $13 \mathrm{~A}$ atoms inside a RSS of concentration $c$ ) or for $\mathrm{Z}=0 \mathrm{~A}$ atoms, which means $12 \mathrm{~B}$ neighbors for a $\mathrm{B}$ atom (a cluster of $13 \mathrm{~B}$ atoms in the same RSS). This dependence on concentration comes essentially from elastic effects; therefore, we called it $\Delta H_{\text {Size }}^{\text {mixing }}(c)$.

Beside the size contribution, the Ising and triplets terms represent the chemical contribution: $\Delta H_{\text {Chem }}^{\text {mixing }}(c)=$ $\Delta H_{\text {Ising }}^{\text {mixing }}(c)+\Delta H_{\text {triplets }}^{\text {mixing }}(c)$. 


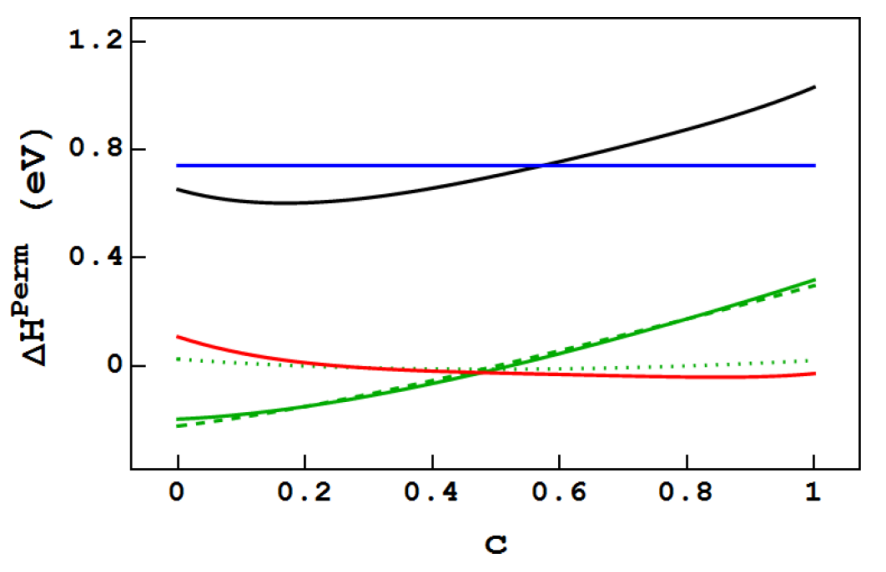

FIG. 8. $\Delta H_{\mathrm{RSS}}^{\mathrm{perm}}$ evolution as a function of the concentration (in black) and decomposition of the different contributions: $\Delta H_{\mathrm{Coh}}^{\mathrm{perm}}$ (in blue), $\Delta H_{\text {Size }}^{\text {perm }}$ (in red), $\Delta H_{\text {Chem }}^{\text {perm }}$ (in green), $\Delta H_{\text {Ising }}^{\text {perm }}$ (dashed green), and $\Delta H_{\text {triplets }}^{\text {perm }}$ (dotted green) with $\Delta H_{\text {Chem }}^{\text {perm }}=\Delta H_{\text {Ising }}^{\text {perm }}+\Delta H_{\text {triplets }}^{\text {perm }}$.

To get the permutation enthalpy we derive either the Hamiltonian or the mixing enthalpy:

$$
\Delta H_{\mathrm{RSS}}^{\mathrm{perm}}(c)=\frac{\partial H(c)}{N_{\mathrm{at}} \partial c}=\frac{\partial \Delta H_{\mathrm{RSS}}^{\text {mixing }}(c)}{\partial c}+\left[E_{A}^{Z}(1)-E_{B}^{0}(0)\right] .
$$

Additionally, we decompose the permutation enthalpy according to the "three effects rule" into

$$
\Delta H_{\mathrm{RSS}}^{\mathrm{perm}}(c)=\Delta H_{\mathrm{Coh}}^{\mathrm{perm}}(c)+\Delta H_{\mathrm{Chem}}^{\mathrm{perm}}(c)+\Delta H_{\mathrm{Size}}^{\mathrm{perm}}(c),
$$

where $\Delta H_{\mathrm{Coh}}^{\mathrm{perm}}(c), \Delta H_{\mathrm{Chem}}^{\mathrm{perm}}(c)$, and $\Delta H_{\text {Size }}^{\mathrm{perm}}(c)$ represent, respectively, the cohesive, the chemical, and the size effects, which are detailed in Appendix D.

\section{Driving forces for the Au-Pd system}

Figure 8 emphasizes the permutation enthalpy in RSS and its reconstitution in terms of cohesive, chemical, and elastic contributions. This distinguishes clearly the origin of

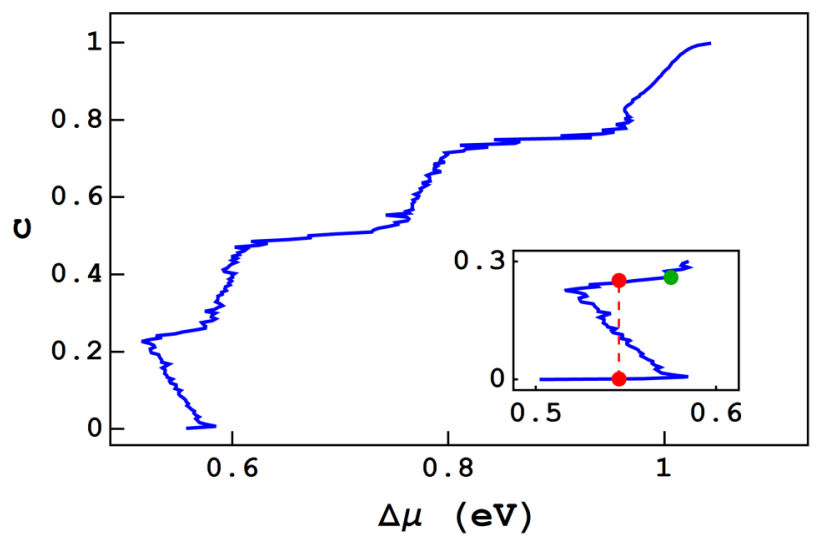

(a) the chemical and elastic driving forces taking place in phase diagrams [5].

We notice in Fig. 8 that $\Delta H_{\mathrm{RSS}}^{\text {perm }}(c)$ has a nonmonotonic character, similarly to $\Delta H_{\mathrm{SMA}}^{\text {perm }}(c)$ (Fig. 3 ), whereas the classical model of solid solution provides a linear and increasing evolution of $\Delta H_{\mathrm{RSS}}^{\mathrm{perm}}$ for a system with ordering tendency like $\mathrm{Au}-\mathrm{Pd}$. While at low concentrations the slope of $\Delta H_{\mathrm{RSS}}^{\mathrm{perm}}(c)$ is negative, it becomes positive for $c \gtrsim 0.2$. In fact, as the gold concentration increases, the permutation of a $\mathrm{Pd}$ atom into an $\mathrm{Au}$ atom becomes easier and easier for the low concentrations and more and more difficult for the high concentrations. Within an Ising model, the slope of $\Delta H_{\mathrm{RSS}}^{\text {perm }}(c)$ is linked to the alloy interaction, which means the tendency to form homo- or heteroatomic bonds [Eq. (C3a)]. Thus, without the decomposition, we could have supposed that the Au-Pd alloy presents a demixtion tendency for the Au-poor systems and ordering tendency for the Au-rich systems. The driving forces analysis developed within the effective site energy model shows that the situation is different. The chemical contribution presents an increasing monotonic evolution in the whole concentration range, consistent with the tendency to form heteroatomic pairs. It is worth noticing that the chemical effect is practically fully due to effective pair interactions $\left(\Delta H_{\mathrm{Ising}}^{\text {perm }}\right)$, the slope of the triplet term $\Delta H_{\text {triplets }}^{\text {perm }}$ being relatively negligible.

The origin of the negative slope of $\Delta H_{\mathrm{RSS}}^{\mathrm{perm}}$ as a function of $c$ for $c<0.2$ is essentially due to the size effect. Indeed, as we can see in Fig. 8 , the slope of $\Delta H_{\text {Size }}^{\text {perm }}$ is negative in a large concentration range, starting from pure $\mathrm{Pd}$, and becomes near to zero when $c$ tends to 1. Elastic and chemical effects are in competition, mainly in the low Au concentration range. For $0 \leqslant c \lesssim 0.2$ the slope of the size effect is predominant and leads to a negative slope for $\Delta H_{\mathrm{RSS}}^{\text {perm }}$, whereas for $c \gtrsim 0.2$ the slope of the chemical effect prevails, leading to a positive slope for $\Delta H_{\mathrm{RSS}}^{\mathrm{perm}}$.

\section{Au-Pd PHASE DIAGRAM $(c \in[0,0.25])$}

To analyze further the competition between elastic and chemical effects, we detail the phase diagram of the system $\mathrm{Au}_{c} \mathrm{Pd}_{1-c}$ in the range $c<0.25$. The canonical isotherm at

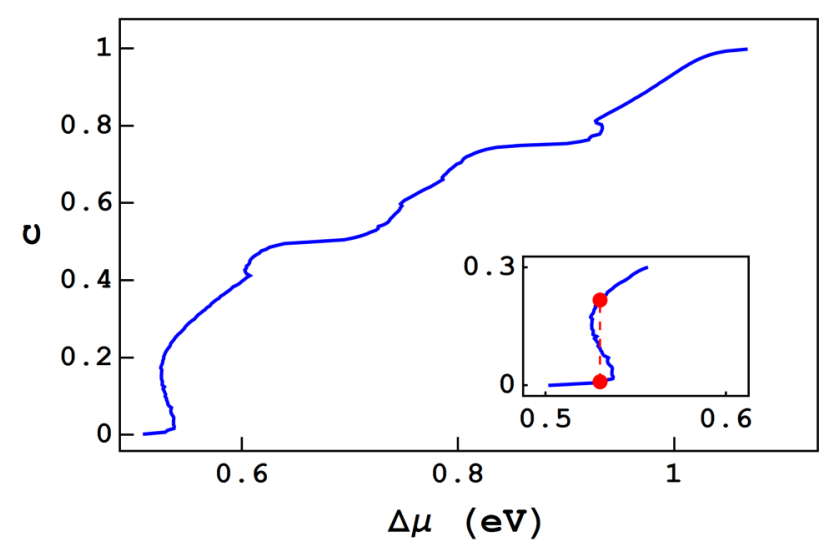

(b)

FIG. 9. $c(\Delta \mu)$ isotherms within Monte Carlo SMA simulations in canonical ensemble at $T=100 \mathrm{~K}$ (a) and at $175 \mathrm{~K}$ (b). The insets represent the isotherms in the concentration range [0,0.3]. Red and green dots represent the solubility limits, respectively, due to elastic effect and chemical effect. 


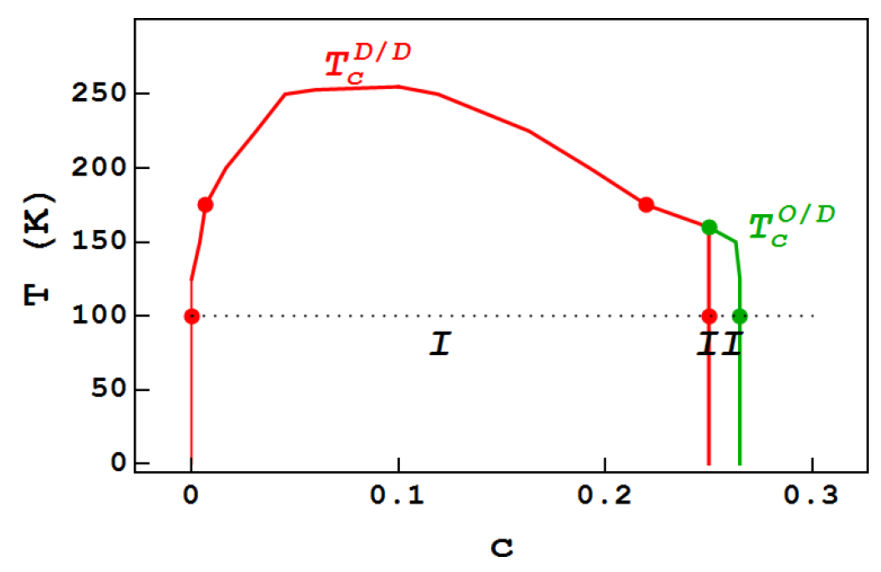

FIG. 10. Phase diagram for $c \in[0,0.3]$. The colors recall the predominant driving forces: chemical effect (green) and size effect (red). I, biphase domain; II, $L 1_{2}$ ordered domain.

$100 \mathrm{~K}$ indicates the presence of an " $S$ " loop up to $c=0.25$, followed by a plateau at $c=0.25$ [Fig. 9(a)]. This plateau characterizes the stability of the $L 1_{2}$ phase. This phase is expected for a positive first-neighbors effective pair interaction. We also notice two others plateaus at concentrations around 0.50 and 0.75 corresponding to the AuPd $L 1_{0}$ and $\mathrm{Au}_{3} \mathrm{Pd} L 1_{2}$ ordered phases. Here we are interested in the $S$ loop below $c=0.25$ [insets in Figs. 9(a) and 9(b)] and in particular its surprisingly large amplitude which implies a quite extended biphase domain in the phase diagram between an almost Pd-pure solid solution and the ordered $\mathrm{AuPd}_{3}$ phase via the equal areas rule. This contrasts strongly with the very restricted coexistence domain predicted by the Ising model, the solubility limit reaching almost $20 \%$ of $\mathrm{Au}$ in Pd. Even more astonishing is the isotherm at $175 \mathrm{~K}$ [Fig. 9(b)]: the $S$ loop remains whereas the plateau at $c=0.25$ has disappeared. This implies that the critical temperature of the $L 1_{2}$ phase lies between 100 and $175 \mathrm{~K}$. The persistence of the $S$ loop at $175 \mathrm{~K}$, although the $L 1_{2}$ phase is vanished, shows that the phase coexistence and the equal areas rule have no relation with the $L 1_{2}$ ordered phase which is observed at very low temperature.

The driving forces analysis explains this unexpected behavior. At $c=0.25$, the order/disorder transition is mainly driven by the chemical effect (predominance of the pair interaction as compared to the triplets ones) and the critical order/disorder temperature $T_{c}^{O / D}$ value is around $160 \mathrm{~K}$. On the other hand, between $c=0$ and $c \approx 0.25$, the $S$ loop is due to the negative slope of the permutation enthalpy in this concentration range and therefore to the size effect. The "demixtion/disorder" transition temperature $T_{c}^{D / D}$ has thus no reason to be equal to the critical order/disorder temperature $T_{c}^{O / D}$ and it is necessary to reach $T_{c}^{D / D}=250 \mathrm{~K}$ to see the disappearance of the $S$ loop.

The bulk phase diagram for gold concentration lower than $30 \%$ and temperature between 0 and $300 \mathrm{~K}$ is illustrated in Fig. 10. The diversity of the driving forces highlighted in this paper explain why this phase diagram looks unusual.

Up to the critical temperature of the $L 1_{2}$ phase $\left(T_{c}^{O / D}=\right.$ $160 \mathrm{~K}$ ), it is characterized by a very large biphase domain (domain I in Fig. 10) of elastic origin almost pure in $\mathrm{Pd}$ $(c \approx 0)$ and an ordered phase around $25 \%$ of gold, the limit concentrations being almost athermal. The chemical effect which controls the ordering of the $\mathrm{AuPd}_{3}$ compound allows only a slight out-of-stoichiometry difference for the $L 1_{2}$ ordered phase (domain II in Fig. 10).

Between the order/disorder temperature $(160 \mathrm{~K})$, controlled by the chemical effect, and the demixtion/disorder temperature $(250 \mathrm{~K})$, controlled by the elastic effect, the phase coexistence, of elastic origin, takes place between a Pd-rich solid solution (almost pure in $\mathrm{Pd}$ since $c$ is only varying from 0 to 0.1 in this temperature range) and another solid solution the concentration of which evolves from 0.25 to 0.1 by increasing the temperature. Above $T_{c}^{D / D}$, the phase diagram displays only a solid solution in the whole concentration range.

\section{CONCLUSION}

We have shown that the interplay of chemical, size, or vibrational driving forces underlies the complex effects on the phase diagram. Accurate analysis is required to identify and account for each contribution. Using original methodology, we succeed in separating unambiguously the elastic component from the chemical one using the permutation enthalpy, a key quantity to characterize the phase diagrams, and also the entropic component from the enthalpic one by considering the permutation free enthalpy.

Applied to the Au-Pd alloy, this approach allows one to understand the origin of a particular topology of the phase diagram with a persistence of a biphased domain between two solid solutions: a very Pd-rich one and another one, less rich in Pd (10 to $25 \%$ of $\mathrm{Au}$ ), above the critical order/disorder temperature of the $\mathrm{AuPd}_{3} L 1_{2}$ ordered phase. We showed that the phase coexistence is essentially due to elastic effects, whereas the ordering of the $\mathrm{AuPd}_{3}$ phase has essentially a chemical origin. This competition between elastic and chemical effects is rather general and therefore should be observed in other alloys, especially in alloys with a weak ordering tendency (with low critical temperature) and with a large lattice misfit. This biphase domain will appear on the side of the phase diagram where the solute atoms are biggest, like in the Au-Pd system. We hope that this paper will stimulate experimental work at low temperatures for these alloys, if necessary using irradiation to overcome kinetic blockages.

More generally, the present exhaustive approach considering the permutation enthalpy and its decomposition in chemical, elastic, and cohesive components (this last one being independent of $c$, it does not contribute to the bulk phase diagram) opens many avenues in the field of phase diagrams. Constantly increasing computing power gives access to many other energetic models and consequently the site energies become more accessible in more sophisticated energetic models, beyond semiempirical potentials SMA, such as tight-binding formalisms or ab initio methods. The question is still open for $a b$ initio calculations for which the site energies are not so well defined.

\section{ACKNOWLEDGMENT}

The authors would like to thank H. Guesmi, B. Zhu, and G. Tréglia for very fruitful discussions. 
TABLE I. Parameters of the Au-Pd SMA potential.

\begin{tabular}{lcccccc}
\hline \hline $\mathrm{I}-\mathrm{J}$ & $E_{\mathrm{coh}}(\mathrm{eV})$ & $a(\AA)$ & $A_{I J}(\mathrm{eV})$ & $p_{I J}$ & $q_{I J}$ & $\xi_{I J}(\mathrm{eV})$ \\
\hline $\mathrm{Pd}-\mathrm{Pd}$ & -4.98 & 3.85 & 0.14352 & 11.75280 & 2.87486 & 1.88570 \\
$\mathrm{Au}-\mathrm{Au}$ & -4.24 & 4.08 & 0.22043 & 10.71890 & 4.09805 & 1.97573 \\
$\mathrm{Au}-\mathrm{Pd}$ & & & 0.21410 & 11.23585 & 3.48645 & 2.08060 \\
\hline \hline
\end{tabular}

\section{APPENDIX A: NUMERICAL METHODS}

\section{SMA interatomic potentials}

We use $N$-body interatomic potentials derived from the SMA of the tight-binding scheme [31,32]. These potentials have been shown to be quite successful to study bulk, surface, and grain boundaries in metallic alloys [33-37]. We just recall here that the potential energy at each site $i$ is written as

$$
\begin{aligned}
E_{i}= & \sum_{j \neq i} A_{I J} \exp \left[-p_{I J}\left(\frac{r_{i j}}{r_{I J}^{0}}-1\right)\right] \\
& -\sqrt{\sum_{j \neq i} \xi_{I J}^{2} \exp \left[-2 q_{I J}\left(\frac{r_{i j}}{r_{I J}^{0}}-1\right)\right]},
\end{aligned}
$$

where $r_{i j}$ is the distance between atoms at sites $i$ and $j$, respectively, occupied by the $I$ and $J$ chemical species $(I, J=$ $\mathrm{Au}, \mathrm{Pd})$, and $r_{I I}^{0}$ is the first-neighbor distance in the metal $I\left(r_{I J}^{0}=\left(r_{I I}^{0}+r_{J J}^{0}\right) / 2\right)$. The interactions in Eq. (A1) are evaluated up to a distance $r_{t}$. To avoid discontinuities in both energy and force, the exponentials are connected to zero with a fifth-order polynomial between $r_{t}$ and the cutoff distance of the potential $r_{c}$. Here, we choose $r_{t}=\max \left(\sqrt{2} r_{I I}^{0}, \sqrt{2} r_{J J}^{0}\right)$ and $r_{c}=\min \left(2 r_{I I}^{0}, 2 r_{J J}^{0}\right)$. The three sets of the four parameters $\left(A_{I J}, p_{I J}, q_{I J}, \xi_{I J}\right)$ listed in Table I are the same as those of Ref. [38]. They have been fitted to the values of the cohesive energies, lattice parameters, (001) and (111) surface energies for pure metals, and mixing energies in the diluted limits calculated using the density functional theory with the local-density approximation for the exchange and correlation functional $[39,40]$.

\section{Site energies}

We use the SMA potentials described above to compute the site energies $E_{I}^{p}(c)$, i.e., the energies of atoms $I(I=\mathrm{A}, \mathrm{B})$, as a function of the number $p$ of $\mathrm{A}$ nearest neighbors in a relaxed RSS of a $\mathrm{A}_{c} \mathrm{~B}_{1-c}$ binary alloy [5]. Note that only the nearest-neighbor shell is considered here but the ESE model can be extended to more distant shells of neighbors if necessary. We use the FIRE algorithm [41] to minimize the potential energy of the RSS until the temperature reaches $10^{-6} \mathrm{~K}$. The simulation box is constructed with seven fcc cubic cells in each direction $\left(N_{\text {at }}=1372\right.$ atoms $)$ and threedimensional periodic boundary conditions. The volume of the simulation box is optimized for each concentration $c$ by searching iteratively the minimum of the $E-V$ curve, the geometry of the simulation box being kept cubic. Then the procedure can be described as follows.

(1) A site $I$ is selected, and its chemical nature is fixed $(I=\mathrm{A}, \mathrm{B})$.
(2) The local environment of $I$ is constructed randomly with $p$ nearest neighbors being $\mathrm{A}$ atoms, the remaining $12-p$ sites being filled with $\mathrm{B}$ atoms.

(3) The remaining $N_{\text {at }}-13$ sites are filled randomly with $\mathrm{A}$ and $\mathrm{B}$ atoms to reach the nominal concentration $c$.

For a given RSS, the $E_{I}^{p}(c)$ are averaged over all the existing configurations surrounding atoms of type $I$ that correspond with the local p environment in A atoms. Finally, the procedure is repeated 100 times for each local $p$ environment to obtain good statistics over the $E_{I}^{p}(c)$. The standard deviation, which depends on the concentration and $p$ values, is less than $5 \times 10^{-3} \mathrm{eV}$.

\section{Numerical simulations}

We proceed with two types of numerical simulations, one to account only for temperature effect on structural relaxation of the RSS binary alloy and the other one to account both for temperature effect on structural relaxation and for repartition of the constituents of a $\mathrm{A}_{c} \mathrm{~B}_{1-c}$ alloy.

The first one allows us to determine the total energy of a RSS binary alloy $E_{\mathrm{RSS}}^{\text {tot }}(c)$ at concentration c and temperature $T$. $E_{\mathrm{RSS}}^{\mathrm{tot}}(c)$ is determined in the whole range of concentration by MC simulations using a standard Metropolis algorithm in which only random atomic displacements and variations of the lattice parameter in the three directions are proposed. The averages are evaluated over $1 \times 10^{5}$ MC macrosteps, a MC macrostep corresponding to $N_{\text {at }}$ moves of random atomic displacement and one move of lattice-parameter variation. For each couple $(T, c)$, we calculate the total energy for the 100 RSS binary solutions used for the determination of the site energies.

The second one allows us to determine the total energy of the equilibrium state $E_{\mathrm{SMA}}^{\text {tot }}(c)$ and the equilibrium chemical potential difference $\Delta \mu=\mu_{A}-\mu_{B}$ as a function of both the concentration and the temperature by means of MC simulations with atomic relaxations in the canonical ensemble. $\Delta \mu(c)$ is obtained via the Widom method [42]. As previously, a standard Metropolis algorithm is used and $\Delta \mu$ is evaluated over $1 \times 10^{5} \mathrm{MC}$ macrosteps, here a macrostep being the same as before plus $N_{\text {at }}$ moves of exchange between heteroatomic pairs of atom.

\section{Vibrational properties}

To obtain the vibrational contribution to the permutation entropy in harmonic approximation we use either the FIRE algorithm or Monte Carlo simulations at low temperature with only random atomic displacements and variations of the lattice parameter. We compute the phonon density of states (PDOS), $n(v)$, directly by diagonalization of the dynamical matrix evaluated in the harmonic approximation [43]. The vibrational entropy is then obtained from the PDOS by

$$
\begin{aligned}
S_{\mathrm{vib}}^{\mathrm{tot}}= & k_{B} \int_{0}^{v_{\max }}\left\{\frac{h v}{2 k_{B} T} \operatorname{coth}\left(\frac{h v}{2 k_{B} T}\right)\right. \\
& \left.-\ln \left[2 \sinh \left(\frac{h v}{2 k_{B} T}\right)\right]\right\} n(v) d v,
\end{aligned}
$$

where $h$ and $k_{B}$ are, respectively, Planck's constant and Boltzmann's constant. 
From the total energies $E_{\mathrm{RSS}}^{\text {tot }}(c)$ or $E_{\mathrm{SMA}}^{\text {tot }}(c)$, one can get directly the mixing enthalpy:

$$
\begin{aligned}
\Delta H_{X}^{\text {mixing }}(c) & =\left\{E_{X}^{\mathrm{tot}}(c)-\left[c E_{X}^{\mathrm{tot}}(1)+(1-c) E_{X}^{\mathrm{tot}}(0)\right]\right\} / N_{\mathrm{at}}, \\
X & =\mathrm{RSS}, \mathrm{SMA},
\end{aligned}
$$

whereas the vibrational contribution of the mixing entropy is deduced from $S_{\text {vib }}^{\text {tot }}$ :

$$
\Delta S_{\mathrm{vib}}^{\mathrm{mixing}}(c)=\left\{S_{\mathrm{vib}}^{\mathrm{tot}}(c)-\left[c S_{\mathrm{vib}}^{\mathrm{tot}}(1)+(1-c) S_{\mathrm{vib}}^{\mathrm{tot}}(0)\right]\right\} / N_{\mathrm{at}} .
$$

In the limit of high temperature, all the phonon modes are excited and the above quantity is almost a constant for temperatures higher than the Debye temperature $\left(T_{D}^{\mathrm{Au}}=170 \mathrm{~K}\right.$ and $\left.T_{D}^{\mathrm{Pd}}=275 \mathrm{~K}\right)$ and we choose its value at $T=600 \mathrm{~K}$ in this work.

\section{APPENDIX B: CONTRIBUTION TO LOCAL ORDER}

The entropy linked to the local order can be expressed from pair probabilities formalism $[28,29]$ :

$$
S_{\mathrm{SRO}} / N_{\mathrm{at}}=-k_{B} Z\left(-\sum_{I=A, B} P_{I} \ln P_{I}+\frac{1}{2} \sum_{I, J} P_{I J} \ln P_{I J}\right),
$$

With $P_{A}=c, P_{B}=1-c, P_{A A}=c^{2}+c(1-c) \eta, P_{A B}=$ $P_{B A}=c(1-c)(1-\eta)$, and $P_{B B}=(1-c)^{2}+c(1-c) \eta$, where $\eta$ is the Warren-Cowley parameter [30].

The permutation entropy linked to the local order, $\Delta S_{\mathrm{SRO}}^{\mathrm{perm}}$, is obtained by the derivation of $S_{\mathrm{SRO}}$ :

$$
\Delta S_{\mathrm{SRO}}^{\mathrm{perm}}=-k_{B} Z\left(-\sum_{I=A, B} P_{I}^{\prime} \ln P_{I}+\frac{1}{2} \sum_{I, J} P_{I J}{ }^{\prime} \ln P_{I J}\right),
$$

with $\quad P_{A}{ }^{\prime}=1, \quad P_{B}^{\prime}=-1, \quad P_{A A^{\prime}}=2 c+(1-2 c) \eta+$ $c(1-c) \frac{\partial \eta}{\partial c}, \quad P_{A B}^{\prime}=P_{B A}^{\prime}=(1-2 c)(1-\eta)-c(1-c) \frac{\partial \eta}{\partial c}$, and $P_{B B}^{\prime}=-2(1-c)+(1-2 c) \eta+c(1-c) \frac{\partial \eta}{\partial c}$.

\section{APPENDIX C: CONTRIBUTION OF THE TRIPLETS}

In a rigid lattice formalism, the energy of the system can be written as a sum of interactions between pairs, triplets, etc. of atoms:

$H=\frac{1}{2} \sum_{I J} \sum_{n, m \neq n} p_{n}^{I} p_{m}^{J} V_{n m}^{I J}+\frac{1}{6} \sum_{I J K} \sum_{l, n, m} p_{n}^{I} p_{m}^{J} p_{l}^{K} V_{n m l}^{I J K}+\ldots$

where $p_{n}^{I}$ represents the occupation factor of the site $n$ : it is equal to 1 if the site $n$ is occupied by an atom of type $I$ $(I=\mathrm{A}, \mathrm{B})$ or zero if not; $V_{n m}^{I J}$ (respectively, $\left.V_{l n m}^{I J K}\right)$ is the pair (respectively, triplets) interaction energy between an atom of type $I$ on a site $n$ and an atom of type $J$ on a site $m(I, J=\mathrm{A}$, B) [respectively, and an atom of type $K$ on a site $l(I, J, K=$ A, B)].

The mean-field approximation is fully justified to describe a disordered solid solution, which leads to

$$
\langle H\rangle=\langle H\rangle_{\text {pairs }}+\langle H\rangle_{\text {triplets }}+\ldots,
$$

with

$$
\begin{aligned}
\langle H\rangle_{\text {pairs }} / N_{\text {at }}= & \frac{Z}{2}\left[c^{2}\left(V_{A A}+V_{B B}-V_{A B}-V_{B A}\right)\right. \\
& \left.+c\left(-2 V_{B B}+V_{A B}+V_{B A}\right)+V_{B B}\right] \\
= & Z c\left(V_{A A}-V_{B B}\right) / 2-Z c(1-c) \\
& \times\left(V_{A A}+V_{B B}-V_{A B}-V_{B A}\right) / 2+Z V_{B B} / 2
\end{aligned}
$$

where $\left(V_{A A}+V_{B B}-V_{A B}-V_{B A}\right) / 2$ is the alloy pair interaction $V_{1}, Z\left(V_{A A}-V_{B B}\right) / 2$ is the difference of cohesive energies between $A$ and $B$, and $Z V_{B B} / 2$ is the B-cohesive energy:

$$
\begin{aligned}
\langle H\rangle_{\text {triplets }} / N_{\mathrm{at}}= & \frac{Z(Z-1)}{2}\left\{c ^ { 3 } \left[\left(V_{A A A}-V_{B B B}\right)\right.\right. \\
& \left.+3\left(V_{B B A}-V_{A A B}\right)\right] \\
& +3 c^{2}\left(V_{A A B}+V_{B B B}-2 V_{A B B}\right) \\
& \left.+3 c\left(V_{A B B}-V_{B B B}\right)+V_{B B B}\right\} .
\end{aligned}
$$

We recognize in Eq. (C3) the different terms of Eq. (13a). We can easily show that $\left\langle H_{N-\text { plets }}\right\rangle$ is a polynomial function of order $N$ in $c$.

Practically, the Ising model (pair model) is very often used. However, we can wonder if it is necessary to take into account the triplets, quadruplets, or more contributions. The matching with the site energies allows us to answer this question.

The energy of a disordered solid solution as a function of site energies is written as follows [5]:

$$
H(c) / N_{\mathrm{at}}=\sum_{p=0}^{Z} C_{Z}^{p} c^{p}(1-c)^{Z-p}\left[c E_{A}^{p}(c)+(1-c) E_{B}^{p}(c)\right],
$$

where the site energies can be written as a sum of a linear function of the number $p$ of first neighbors atoms of A type and a complementary term $\Delta E_{X}^{p}(c)$ :

$$
E_{X}^{p}(c)=E_{X}^{0}(c)+\frac{p}{Z}\left[E_{X}^{Z}(c)-E_{X}^{0}(c)\right]+\Delta E_{X}^{p}(c) .
$$

$\Delta E_{X}^{p}(c)$ represents the nonlinear part of $E_{X}^{p}(c)$ as a function of $p . \Delta E_{X}^{p}(c)$ is written as a polynomial function:

$$
\Delta E_{X}^{p}(c)=\varkappa_{X} p^{m}(Z-p),
$$

where $\varkappa_{X}$ represents the curvature.

When $\Delta E_{X}^{p}(c)=0$, i.e., when the site energies vary linearly with $p$, the energy of the system is a polynomial function of order 2 in $c$ :

$$
\begin{aligned}
H(c) / N_{\mathrm{at}}= & c^{2}\left[E_{A}^{Z}(c)-E_{A}^{0}(c)-E_{B}^{Z}(c)+E_{B}^{0}(c)\right] \\
& +c\left[-2 E_{B}^{0}(c)+E_{B}^{Z}(c)-E_{A}^{0}(c)\right]+E_{B}^{0}(c) .
\end{aligned}
$$

The identification term to describe the polynomial coefficients with those of $\left\langle H_{\text {pairs }}\right\rangle$ leads to the following definitions: $H_{\text {Ising }}(c) / N_{\text {at }}=c Z \alpha(c)-c(1-c) Z V_{1}(c)+$ $Z V_{B B} / 2$ with $Z \alpha(c)=E_{A}^{Z}(c)-E_{B}^{0}(c)$, the alloy pair interactions restricted to the nearest-neighbor shell $Z V_{1}(c)=$ $\left[E_{A}^{Z}(c)-E_{A}^{0}(c)\right]-\left[E_{B}^{Z}(c)-E_{B}^{0}(c)\right]$ and $Z V_{B B} / 2=E_{B}^{0}(c)$ [5]. 
In the present case, $\Delta E_{X}^{p}(c)$ is a polynomial function of order 2 in $p(m=1)$. The energy is then the sum of a linear term [Eq. (13a)] and a complementary term $\Delta H(c)$ :

$$
\Delta H(c)=Z(Z-1) c(1-c)\left[c \varkappa_{A}(c)+(1-c) \varkappa_{B}(c)\right],
$$

which is a polynomial function of order 3 in $c$. The Ising model is no longer sufficient but it is necessary (and sufficient) to take into account the triplets. More generally, when $E_{X}^{p}(c)$ is a polynomial of order $n$ in $p$, it is necessary and sufficient to take into account the interactions of the pairs, triplets, etc., up to the $(n+1)$-plets to describe the energy of a random solid solution.

\section{APPENDIX D: $\Delta H_{\mathrm{RSS}}^{\mathrm{perm}}(c)$ DRIVING FORCES}

We detail in this Appendix the different contributions of the permutation enthalpy of a RSS. The permutation enthalpy is obtained by derivation of the Hamiltonian [Eq. (13)] or of the mixing enthalpy:

$$
\Delta H_{\mathrm{RSS}}^{\mathrm{perm}}=\frac{\partial \Delta H_{\mathrm{RSS}}^{\mathrm{mixing}}(c)}{\partial c}+\left[E_{A}^{Z}(1)-E_{B}^{0}(0)\right]
$$

where the term $E_{A}^{Z}(1)-E_{B}^{0}(0)$ is the difference between the cohesive energy of the two pure metals; we call it the cohesion effect $\Delta H_{\mathrm{Coh}}^{\mathrm{perm}}(c)$.

Therefore we have $\Delta H^{\text {perm }}=\frac{\partial \Delta H_{\text {Chem }}^{\text {mixing }}(c)}{\partial c}+\frac{\partial \Delta H_{\text {Size }}^{\text {mixing }}(c)}{\partial c}+$ $\Delta H_{\text {Coh }}^{\text {perm }}(c)$, with $\Delta H_{\text {Chem }}^{\text {perm }}(c)=\left.\frac{\partial \Delta H_{\text {Chem }}^{\text {mixing }}(c)}{\partial c}\right|_{a}$ and $\Delta H_{\text {Size }}^{\text {perm }}(c)$ $=\left.\frac{\partial \Delta H_{\text {Chem }}^{\text {mixim }}(c)}{\partial c}\right|_{b}+\frac{\partial \Delta H_{\text {Sizi }}^{\text {mixing }}(c)}{\partial c}$ where $\left.\frac{\partial X}{\partial c}\right|_{a}$ means that we derive only the combinatorial terms of the variable $X$ whereas for $\left.\frac{\partial X}{\partial c}\right|_{b}$ we derive only the site energies. This allows one to define the three effects

$$
\begin{gathered}
\Delta H_{\mathrm{Coh}}^{\mathrm{perm}}=E_{A}^{Z}(1)-E_{B}^{0}(0), \\
\Delta H_{\mathrm{Chem}}^{\mathrm{perm}}(c)=\Delta H_{\text {Ising }}^{\text {perm }}(c)+\Delta H_{\text {triplets }}^{\text {perm }}(c),
\end{gathered}
$$

with

$$
\begin{aligned}
\Delta H_{\mathrm{Ising}}^{\text {perm }}(c)= & -Z(1-2 c) V_{1}(c), \quad \text { (D3a) } \\
\Delta H_{\text {triplets }}^{\text {perm }}(c)= & Z(Z-1)\left\{(1-2 c)\left[c \varkappa_{A}(c)+(1-c) \varkappa_{B}(c)\right]\right. \\
& \left.+c(1-c)\left[\varkappa_{A}(c)-\varkappa_{B}(c)\right]\right\}, \quad \text { (D3b) }
\end{aligned}
$$

and

$$
\begin{aligned}
\Delta H_{\text {Size }}^{\text {perm }}(c)= & \left(E_{A}^{Z}(c)-E_{A}^{Z}(1)\right)-\left(E_{B}^{0}(c)-E_{B}^{0}(0)\right)+c\left(\frac{\partial E_{A}^{Z}(c)}{\partial c}\right) \\
& +(1-c)\left(\frac{\partial E_{B}^{0}(c)}{\partial c}\right)-c(1-c)\left(\left(\frac{\partial E_{A}^{Z}(c)}{\partial c}-\frac{\partial E_{A}^{0}(c)}{\partial c}\right)\right. \\
& \left.-\left(\frac{\partial E_{B}^{Z}(c)}{\partial c}-\frac{\partial E_{B}^{0}(c)}{\partial c}\right)\right)+Z(Z-1) c(1-c)\left(c \frac{\partial \varkappa_{A}(c)}{\partial c}+(1-c) \frac{\partial \varkappa_{B}(c)}{\partial c}\right)
\end{aligned}
$$

[1] J. W. D. Connolly and A. R. Williams, Phys. Rev. B 27, 5169(R) (1983).

[2] C. Wolverton, G. Ceder, D. de Fontaine, and H. Dreyssé, Phys. Rev. B 48, 726 (1993).

[3] J. M. Sanchez, F. Ducastelle, and D. Gratias, Physica A 128, 334 (1984).

[4] J. M. Sanchez and J. L. Moran-Lopez, Phys. Rev. B 32, 3534 (1985).

[5] F. Berthier, J. Creuze, and B. Legrand, Phys. Rev. B 95, 224102 (2017).

[6] M. Nishino, P. A. Rikvold, C. Omand, and S. Miyashita, Phys. Rev. B 98, 144402 (2018).

[7] C. H. Chan, G. Brown, and P. A. Rikvold, Phys. Rev. B 96, 174428 (2017).

[8] M. Nishino, S. Miyashita, and P. A. Rikvold, Phys. Rev. B 96, 144425 (2017).

[9] P. A. Rikvold, G. Brown, S. Miyashita, C. Omand, and M. Nishino, Phys. Rev. B 93, 064109 (2016).

[10] W. Nicolazzi, S. Pillet, and C. Lecomte, Phys. Rev. B 78, 174401 (2008).

[11] C. Enachescu, L. Stoleriu, A. Stancu, and A. Hauser, Phys. Rev. Lett. 102, 257204 (2009).

[12] W. Nicolazzi and S. Pillet, Phys. Rev. B 85, 094101 (2012).
[13] H. Spiering, T. Kohlhaas, H. Romstedt, A. Hauser, C. BrunsYilmaz, J. Kusz, and P. Gütlich, Coord. Chem. Rev. 190, 629 (1999).

[14] H. Spiering, Top. Curr. Chem. 235, 171 (2004).

[15] K. Boukheddaden, J. Linares, H. Spiering, and F. Varret, Eur. Phys. J. B 15, 317 (2000).

[16] M. Haruta, N. Yamada, T. Kobayahsi, and S. Lijima, J. Catal. 115, 301 (1989).

[17] H. Okamoto and T. B. Massalski, Bull. Alloy Phase Diagrams 6, 229 (1985).

[18] A. Nagasawa, J. Phys. Soc. Japan 19, 2345 (1964).

[19] A. Nagasawa, Y. Matsuo, and J. Kakinoki, J. Phys. Soc. Japan 20, 1881 (1965).

[20] Y. Matsuo, A. Nagasawa, and J. Kakinoki, J. Phys. Soc. Japan 21, 2633 (1966).

[21] Y. Kawasaki, S. Ino, and S. Ogawa, J. Phys. Soc. Japan 30, 1758 (1971).

[22] S. V. Barabash, V. Blum, S. Muller, and A. Zunger, Phys. Rev. B 74, 035108 (2006).

[23] M. H. F. Sluiter, C. Colinet, and A. Pasturel, Phys. Rev. B 73, 174204 (2006).

[24] J. Kanamori and Y. Kakehashi, J. Phys. Colloq. 38, C7-274 (1977). 
[25] J. Nelayah, N. T. Nguyen, D. Alloyeau, G. Y. Wang, and C. Ricolleau, Nanoscale 6, 10423 (2014).

[26] I. Atanasov and M. Hou, Surf. Sci. 603, 2639 (2009).

[27] B. Shan, L.G. Wang, S. Yang, J. Hyun, N. Kapur, Y. J. Zhao, J. B. Nicholas, and K. Cho, Phys. Rev. B 80, 035404 (2009).

[28] R. Kikuchi, Phys. Rev. 81, 988 (1951).

[29] M. Nastar, HDR thesis, Université Paris Sud, 2010.

[30] C. Wolverton, V. Ozolins, and A. Zunger, J. Phys.: Condens. Matter 12, 2749 (2000).

[31] F. Ducastelle, J. Phys. (Paris) 31, 1055 (1970).

[32] V. Rosato, M. Guillopé, and B. Legrand, Phil. Mag. A 59, 321 (1989).

[33] F. Berthier, B. Legrand, and G. Tréglia, Acta Mater 47, 2705 (1999).

[34] F. Berthier, B. Legrand, and G. Tréglia, Interface. Sci. 8, 55 (2000).

[35] J. Creuze, F. Berthier, R. Tétot, and B. Legrand, Phys. Rev. Lett. 86, 5735 (2001).
[36] F. Berthier, J. Creuze, R. Tétot, and B. Legrand, Applied Surface Science 177, 243 (2001).

[37] R. Tétot, F. Berthier, J. Creuze, I. Meunier, G. Tréglia, and B. Legrand, Phys. Rev. Lett. 91, 176103 (2003).

[38] B. Zhu, A. Front, H. Guesmi, J. Creuze, B. Legrand, and C. Mottet, Computational and Theoretical Chemistry 1107, 49 (2017).

[39] B. Zhu, H. Guesmi, J. Creuze, B. Legrand, and C. Mottet, Phys. Chem. Chem. Phys. 17, 28129 (2015).

[40] J. Creuze, H. Guesmi, C. Mottet, B. Zhu, and B. Legrand, Surface Science 639, 48 (2015).

[41] E. Bitzek, P. Koskinen, F. Gähler, M. Moseler, and P. Gumbsch, Phys. Rev. Lett. 97, 170201 (2006).

[42] B. Widom, J. Chem. Phys. 39, 2808 (1963).

[43] A. A. Maradudin, E. W. Montroll, G. H. Weiss, and I. I. Ipavota, Theory of Lattice Dynamics in the Harmonic Approximation (Academic, New York, 1971). 